\title{
Viscoelastic Behavior of Carpets
}

\author{
Part 2: Experimental Analysis of Factors Influencing the Viscoelastic Behavior
}

By Tsuneo Horino and Shigeo Shimonishi, Members, TMSJ

Department of Textile Engineering, Art Industry and Textile College of Kyoto, Kyoto

Based on the Journal of the Textile Machinery Society of Japan, Transactions, Vol. 24, No. 10, T164-172 (1971)

\begin{abstract}
Looped pile carpets having different pile structure were woven by a tufted machine using Nylon- 6 textured pile yarns. Two different groups of model cut-pile carpets were made by using an attachment; one group consisting of the same apparent density in pile part and the other group of the different apparent density.

Apparent values of complex dynamic modulus have been measured of both the looped pile and the cut pile carpets at various given compressive strains by means of Hysteresis loop method at a fixed frequency of $0.5 \mathrm{~Hz}$. Their complex elastic modulus may be calculated on the assumption that the mechanical model of these carpets is similar to that of Voigt.

The viscoelastic property of the carpets under compression depends on various factors, and especially in this experiments on two factors: deformed mode of a pile yarn and volume fraction of fiber phase in pile part.
\end{abstract}

KEY WORDS: CARPETS, TUFTED CARPETS, DYNAMIC MODULUS, COMPRESSION TESTING, TEXTURED YARN (FILAMENT), VIOGT MODEL, JAMMING, FABRIC FIBER FRICTION

\section{Introduction}

Most experimental studies on compressive kehavior of carpets have been carried out to measure the relationship between compressive load and deformation and find its influence on their end-use. ${ }^{[1-5]}$

In contrast with it, theoretical studies have been done to obtaine the technical data for carpet production by evaluating the introduced force and deformation of a pile yarn during compressive process. ${ }^{[6,7]}$ From the information thus obtained, it will be possible to weave the carpet having desired properties.

Looped pile carpets and model cut pile carpets with different factors affecting visco-elastic behavior were prepared in this experiments.

\section{Modes of Compressive Deformation of Pile Yarns}

Usually, the apparent density in pile part of commercial carpets is approximately $0.12 \mathrm{~g} / \mathrm{cm}^{3}$. Assuming that the pile part is a blend system consisting of both the fiber phase and the open air phase, about $90 \%$ in volume of the pile part is formed by the open air phase as described in later section. Therefore, under soft compression, pile yarns can be deformed freely and show various compresive forces depending on the modes of deformation. Fig. 1 shows schematic diagrams of deformed modes for cut and looped pile yarns under initial compression. When cut pile yarn (a) having apparent diameter $d_{a}$ and pile length $l_{p}$ is subjected to compressive load (1), it will take the three kinds of deformed modes shown in Figs. 1 -(b), (c) and (d). If the value, $l_{p} / d_{a}$, is smaller than 3 , the cut pile yarn may be deformed as shown in Fig. 1-(b), in which the twist angle of the yarn varies but its diameter does not change like a spring coil.

When $l_{p} / d_{a}$ is larger than 5 , the pile yarn deforms like the buckling of a column, whose one end is fixed and the other end is free as shown in Fig. 1-(c). When the yarn axis is not perpendicular to the surface of the base fabric such as seen in flocked carpets, the yarn may be deformed as shown in Fig. 1-(d).

Even if the loop is made of thie same yarn length, a looped pile may be formed with different ratios of loop spacing $S$ to loop length $H$ as shown in Figs. 1-(e) and (g), if the crimp ratio of the yarn is varied due to the difference of crimping processes. Figs. 1-(e) and (f) represent the modes of a looped pile having low ratio of $S / H$ before and after deformation, respectively. At such a high ratio of $S / H$ as shown in Fig. 1-(g), the looped pile may be deformed plain or perpendicular to the plain of the loop, as shown in Figs. 1-(h) and (i), respectively. 


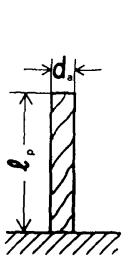

(a)

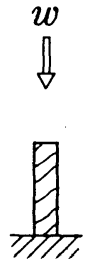

(b)

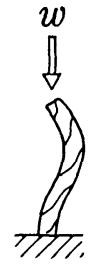

(c)

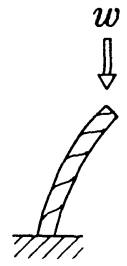

(d)

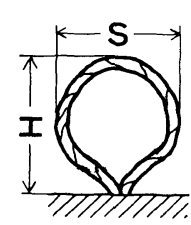

(e)

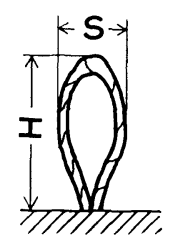

(g)

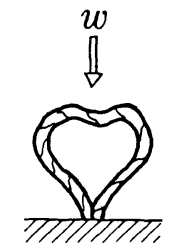

(f)

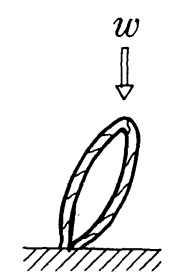

(h)

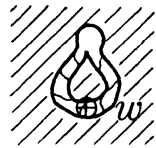

(i)
Fig. 1 Schematic diagrams of compressive deformed modes for cut pile and looped pile yarns

(a): a cut pile yarn having apparent diameter $d_{a}$ and pile length $l_{p}$

(e): a looped pile yarn having high $S / H$ ratio

(g): a looped pile yarn having low $S / H$ ratio

Therefore, compressive modes of the looped pile yarns depend on yarn dimensions and shapes under initial compression. When the compressive strain increases, the carpet structure takes jammed configuration depending on deformed modes, and the fiber phase in pile part reaches the closed packing state. In this case, excepting Fig. 1-(b), the adjoining pile yarns touch each otherduring compression. So, the effect of interaction among pile yarns corresponding to each deformed modes should te considered in the packing state.

Kimura, Kawabata, et al ${ }^{[7]}$ investigated theoretically deformation of the modes as shown in Figs. 1-(c) and (d), A. El-Shiekh, et $a^{\left[{ }^{[6]}\right.}$ also of modes as shown in Figs. 1-(f) and (h).

In the first paper of this series, ${ }^{[8]}$ the dynamic viscoelastic behavior of commercially available carpets was measured. In this study, the tehavior of looped pile carpets made by different processes at various ratios of $S / H$ was measured together with model cut pi'e carpets made with an attachment.

\section{Test Sample}

Carpets have been made of various synthetic fibers, needless to say of natural fibers. Also depending on the manufacturing processes, there are many types different in point of the base structure, the connecting method of the pile part with the base fabric part and so forth.

In this experiments, some samples were prepared with looped pile carpets having different pile structures, but woven on a tufted machine using Nylon- 6 textured yarns. In addition to the looped pipe carpets, model cut pile carpets were made by an attachment since the apparent density in pile part of commercially available carpets is restricted within narrow limits.

\section{3-1 Looped Pile Yarn Carpet Samples}

Looped pile carpet samples were produced by a tufted machine having the loom width of $76 \mathrm{~cm}$, while keeping a constant gauge of $5 / 32$ inch, but changing the number of tufts per inch, in order to obtain approximately the same pile weight for the samples. Pile yarns were tufted into the first base plain fabric made of jute fibers, by changing the number of tufts per inch. And to this tufted fabric the same plain fabric was adhered with latex adhesive as a backing fabric. These looped pile carpets were made by changing the following factors shown in the parenthese.

1) Textured process of pile yarn

-False twist crimping (B)

- Stuffer box crimping. Crimp angles are small (S), middle (M) and large (L).

2) Dyeing process - - Yarn dyed (P)

3) After-treatment - - Untreated made larger

Sample carpets are listed in Table 1 by using these letters. For example, P-SP-M shows the carpet made of middlecrimp-angle yarns, yarn-dyed and after-treated.

Hereafter, the pile weight means the pile yarn weight per $\mathrm{m}^{2}$ before backing treatment, the thickness of carpets $l_{c}$ being such value as when they are compressed with a stress of $4 \mathrm{~g} / \mathrm{cm}^{2}$. Since all carpets were made with the same base fabrics, "pile weight $/ l_{c}$ " is tabulated as the relative value of carpet density.

The constituent fibers in the pile yarn made by the stuffer box crimping were shaped in zigzag configuration, so the mean value of angle at each bending point in zigzag structure is shown in Table 1 as the crimp angle.

To observe the pile structure of these carpets, the sample was first dipt into a molten praffin (m. p. about $60^{\circ} \mathrm{C}$ ) bath set in a vacuum desicator, and the pressure was reduced in order to let the inside of the carpet packed with paraffine. The sample was then cooled to room temperature. They were cut into slices of about $0.3 \mathrm{~mm}$ thickness, perpendicular or parallel to the surface of the base fabric, on a microtome. These slices are shown in Fig. 2.

When textured yarns are not undergone "Utreated" there is little significant difference in loop shape, between 
yarn dyed carpets and carpet dyed samples. But if textured yarns had with the after-treatment, large air gap is found inside of loops as shown in Fig. 2. This is especially clear in case of yarn dyed carpets.

With false twisted yarns, the constituent fibers were distributed uniformely in the loop, as shown in the horizontalcross sections under each figure, which indicates that the fiber distribution of carpet B is the best. These cross sections were obtained at such a position as the loop spacing was roughly maximum.

As shown in Fig. 2, the loop shapes are different with the different processes above mentioned, even if same yarn materials are used. Therefore, compressive deformed modes for these carpets may vary among them as shown in Fig. 1. 3-2 Model Cut Pile Carpet Samples

Since it is difficult to get from commercially available carpets such a sample as having widely different densities in pile part with same pile yarns, model cut pile carpets were made to measure visco-elasticity of pile part alone. This is because the visco-elastic behavior of carpets roughly at $60 \%$ static strain is influenced by the pile part, when a mechanical model of carpet is assumed such that a pile part and a base part are connected in series. ${ }^{[9]}$

Fig. 3 shows an attachment to make the model cut pile yarn carpet. Two combs (5) of the same size are fixed on both edges of a block (1), and a pile yarn (2) is passed through teeth of the combs to arrange it parallel as shown in thin chain line in Fig. 3. Then, the arranged pile yarns are sandwiched with base pieces (3) by using double-side adhesive tape, and are cut into predetermined lengths. The base pieces having pile yarns adhered are then piled up to form a layer. Thereafter, lower sides of these pieces are

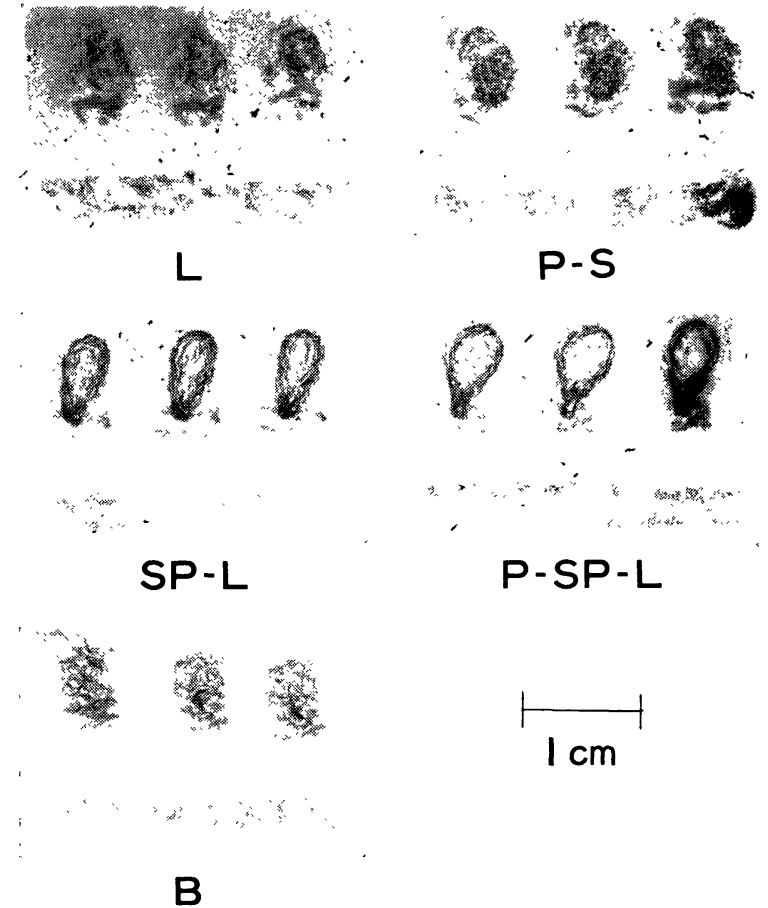

Fig. 2 Enlarged cross sections perpendicular and horizontal to the base fabric surface with five looped carpets

arranged in a plain, and are fixed together, as shown in Fig. 4 , by using bolts through the holes (4).

If the visco-elastic behavior of the carpets are reperesented by the above mentioned mechanical model, the dynamic

Table 1 Mechanical Properties of Looped Pile Carpets

\begin{tabular}{|c|c|c|c|c|c|}
\hline Specifications & $\begin{array}{l}\text { Pile weight } \\
\left(\mathrm{g} / \mathrm{m}^{2}\right)\end{array}$ & $\begin{array}{c}\text { Number of tufts } \\
\text { per inch }\end{array}$ & $\begin{array}{l}\text { Thickness } \\
\text { of carpet } \\
l_{c}(\mathrm{~mm})\end{array}$ & $\begin{array}{l}(\text { pile weight }) / l \\
\left(\mathrm{~g} / \mathrm{cm}^{3}\right)\end{array}$ & $\underset{\left({ }^{\circ}\right)}{\text { Crimp angle }}$ \\
\hline $\mathbf{S}$ & 730 & 11.0 & $9.0_{6}$ & 0.080 & 91.5 \\
\hline M & 702 & 10.5 & $8.9_{2}$ & 0.078 & 104 \\
\hline $\mathrm{L}^{*}$ & 722 & 11.5 & $9.4_{4}$ & 0.076 & 110 \\
\hline $\mathrm{SP}-\mathrm{S}$ & 660 & 10.0 & $9.7_{7}$ & 0.067 & 117 \\
\hline SP-M & 638 & 10.0 & $9.7_{3}$ & 0.065 & 135 \\
\hline $\mathrm{SP}-\mathrm{L}^{*}$ & 758 & 13.0 & $9.8_{0}$ & 0.077 & 150 \\
\hline $\mathrm{P}-\mathrm{S}^{*}$ & 685 & 10.0 & $8.9_{3}$ & 0.076 & 101 \\
\hline $\mathbf{P}-\mathbf{M}$ & 664 & 10.0 & $8.9_{3}$ & 0.074 & 106 \\
\hline $\mathrm{P}-\mathrm{L}$ & 787 & 13.0 & $9.5_{0}$ & 0.082 & 115 \\
\hline P-SP-S & 787 & 12.0 & $9.7_{2}$ & 0.080 & 116 \\
\hline P-SP-M & 705 & 11.0 & $9.7_{3}$ & 0.072 & 127 \\
\hline P-SP-L* & 746 & 12.0 & $9.8_{3}$ & 0.075 & 142 \\
\hline $\mathrm{B}^{*}$ & 586 & 8.5 & $8.6_{2}$ & 0.080 & - \\
\hline
\end{tabular}




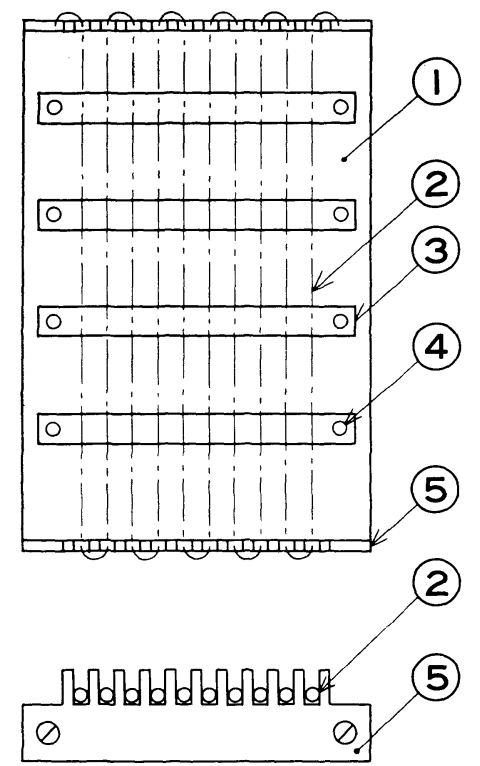

Fig. 3 Schematic diagram of an attachemnt employed for making model cut pile carpets (1): block (2): pile yarn (3): base piece (4): hole for bolt (5): comb

Complex modulus of carpet $E c^{*}$ is given by

$$
\begin{aligned}
& E_{C^{*}}=\left(\begin{array}{c}
\phi_{P} \\
E_{P} *
\end{array} \phi_{B}{ }_{B}^{*}\right)^{-1} \\
& \phi_{P}+\phi_{B}=1 \\
& \phi_{P}=l_{P} / l_{C}, \phi_{B}=l_{B} / l_{C}
\end{aligned}
$$$$
\} \cdots \cdots(1)
$$

where $E_{l}{ }^{*}$ : dynamic complex modulus of a pile part

$E_{B}{ }^{*}$ : dynamic complex modulus of a base part

$\phi_{p}$ : volume fraction of a pile part

$S_{B}$ : volume fraction of a base part

$I_{r}:$ thickness of the carpet

$l_{p}:$ thickness of the pile part

$I_{B}$ : thickness of the base part

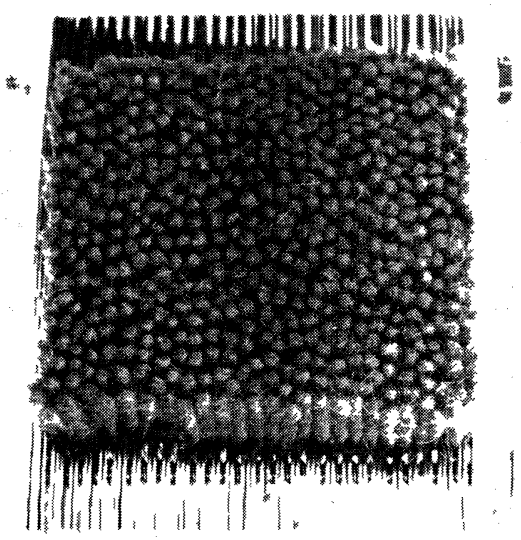

Fig. 4 An example of a model cut pile carpet made of rayon spun pile yarns

From eq. (1), $E p^{*}$ is given by

$$
E_{P^{*}}=\frac{\phi_{P} \cdot E_{c^{*}}}{1-\phi_{B}\left(E_{c^{*}} / E_{B^{*}}\right)}
$$

As seen i t tlic model carpet, if the base fabric is made of metal, which has higher dynamic modulus than fibers, eq. (2) reduces to $\left(2^{\prime}\right)$, tecause $0<\dot{\phi}_{p}<1$ and $0<E c^{*} / E \mathbf{B}^{*} \ll 1$,

$$
E_{P}^{*} \div \phi_{P} \cdot E_{c}^{*}=\left(l_{P} / l_{c}\right) \cdot E_{c}^{*}
$$

Therefore, the visco-elastic behavior of the pile part alone can te obtained by measuring the model carpet.

Model cut pile carpets prepared by the above mentioned procedures with planned density variation, and fiker materials are listed in Table 2.

The pile yarns of model carpets stand approximately

\begin{tabular}{|c|c|c|c|c|c|}
\hline Specifications & Kind of pile yarns & $\begin{array}{l}\text { Thickness of } \\
\text { pile part } \\
l_{p}(\mathrm{~mm})\end{array}$ & $\begin{array}{c}\text { Apparent } \\
\text { density of pile } \\
\text { part } \\
\left(\mathrm{g} / \mathrm{cm}^{3}\right)\end{array}$ & $\begin{array}{l}\text { Density of } \\
\text { fibers } \\
\rho_{f}\left(\mathrm{~g} / \mathrm{cm}^{3}\right)\end{array}$ & $\begin{array}{l}\text { Volume frac. } \\
\text { of pile part } \dot{\phi}_{f}\end{array}$ \\
\hline $\mathbf{M}-\mathbf{A}$ & $\begin{array}{l}\text { Nylon- } 6 \text { textured yarn, } \\
\text { false twist crimped }\end{array}$ & 10.0 & $0.14_{0}$ & 1.14 & $0.12_{3}$ \\
\hline M-B & $\begin{array}{l}\text { Nylon-6 textured yarn, } \\
\text { stuffer box crimped }\end{array}$ & 10.0 & $0,14_{0}$ & 1.14 & $0.12_{3}$ \\
\hline M-R & Rayon spun yarn & 10.0 & $0.14_{0}$ & 1.51 & $0.09_{2}$ \\
\hline M-PP-1 & \multirow{4}{*}{$\begin{array}{l}\text { Polypropylene textured } \\
\text { yarn, stuffer box } \\
\text { crimped }\end{array}$} & 10.0 & $0.14_{0}$ & 0.93 & $0.15_{1}$ \\
\hline M-PP-2 & & 10.0 & $0.10_{0}$ & 0.93 & $0.10_{8}$ \\
\hline M-PP-3 & & 10.0 & $0.05_{8}$ & 0.93 & $0.06_{3}$ \\
\hline M-PP-4 & & 10.0 & $00.2_{3}$ & 0.93 & $0.02_{4}$ \\
\hline
\end{tabular}
perpendicular to the upper surface of the base pieces. Since pile yarns in all model-carpets are cut in $10 \mathrm{~mm}$ length, their deformed mode will be such as shown in Fig. 1-(c).

Table 2 Mechanical Proper iss of Model Cut Pile Carpets and Density of Fibers Used 


\section{Observation of Compressive Deformed State of Pile Part}

Fig. 5 shows the compressive deformed state of three characteristic looped pile carpets se'ected from those listed in Tab'e 1. These observations are carried out by using a small scale compressive tester ${ }^{[9]}$ with a square compressive plate of $2 \times 2 \mathrm{~cm}$

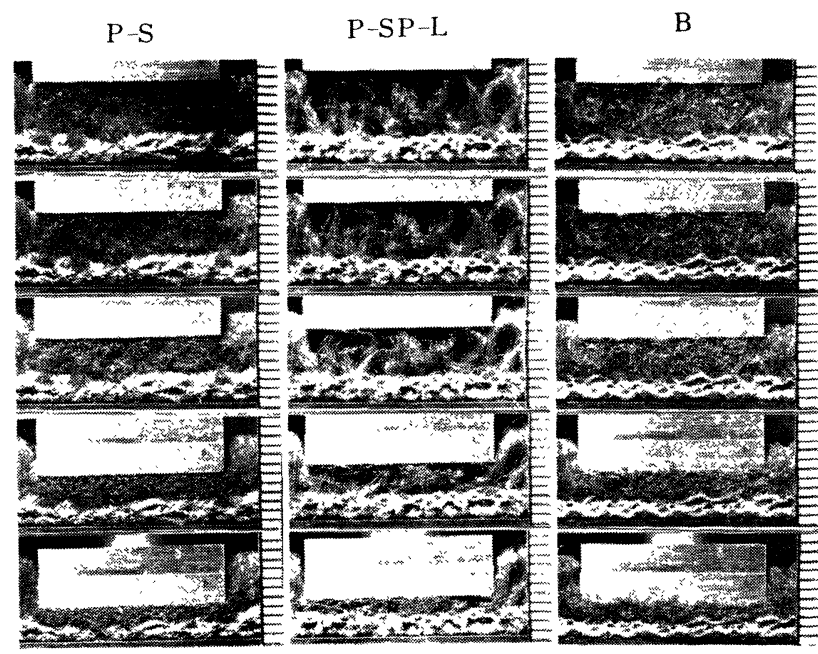

Fig. 5 Compressive deiormed states of three looped pile carpets. One division of the scales at right side represents $1 \mathrm{~mm}$. The strain $\hat{\varepsilon}_{r}$ is $0 \%$. $10 \%, 20 \%, 35 \%$ and $55 \%$ from up to down respestively

As can be seen in Fig. 5, P-S sample and B sample both having high $\mathrm{S} / \mathrm{H}$ ratio may deform as shown in Figs. $1-(\mathrm{h})$ and 1-(i), respectively. For P-SP-L samp!e having low $\mathrm{S} / \mathrm{H}$ ratio, though the arrangement of looped piles become irregulare as the maximum value of $S$ is wider than $5 / 32$ inch, (the gauge length of the tufting machine), the loop may deform as shown in Fig. 1-(f).

When loop shapes at $\varepsilon_{c}=0 \%$ vary as shown in Fig. 5, the jamming configuration of the looped yarn piles may be different as the compressive strain increases. When P-SP-L sample is subjected to compressive strain at $\varepsilon_{c}=35 \%$, each looped pile still holds comparatively large air gap.

On the other hand, P-S and B samples have no such air gap in deformed state at the same strain. Therefore, it should be noted that the degree of compressive force and the external frictional force between fiters are affected by the shapes of looped piles before deformation and by the difference of deformed modes.

The deformed state of a model carpet made of polypropylene yarns are shown in Fig. 6. With the carpets having the same apparent density in pile part, the volume fraction of fiber phase in pile part tecomes the highest, the density of constituent fibers of this carpet being the lowest.

Fig. 6 shows deformed states of a model carpet having

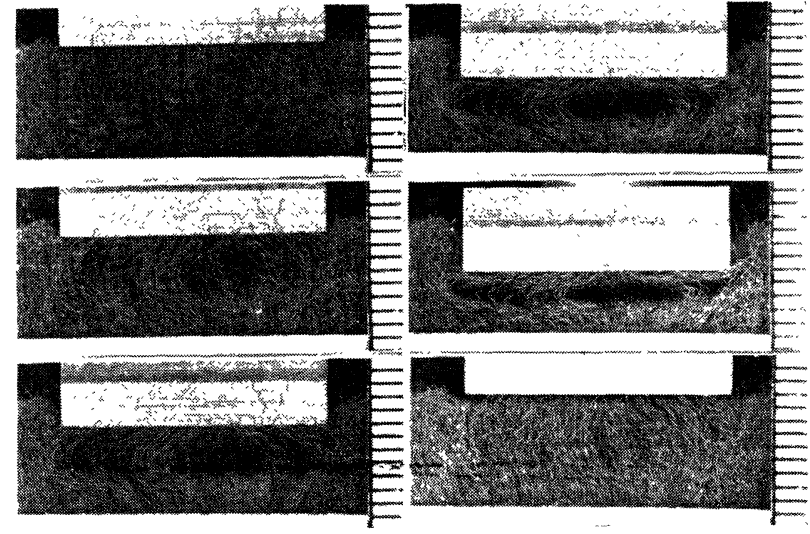

Fig. 6 Compressive deformed states of a model cutpile carpet made of polypropylene pile yarn. One division of the scales at their right hand represents $1 \mathrm{~mm}$. The strain is $0 \%, 10 \%, 20 \%, 30 \%, 45 \%$ and load-removed state from the top of the left column downwards then from the top of the right column downwards

the same value of $\rho_{\imath, \mu}$ as M-PP-1 but with shorter pile length of $8.7 \mathrm{~mm}$ than M-PP-1. Even in this case, the deforned mode takes such as shown in Fig. 1-(c), which shows that air gaps exist under as high strain as $s_{i} \fallingdotseq 45 \%$.

\section{Experimental Results and Discussion}

The dynamic experiment of test carpets was carried out by Hystresis loop method with an apparatus descrited in the previous paper, and the apparent complex dynamic modulus was calculated on the assumption that the mechanical model of a carpet is similar to that of Viogt. ${ }^{[8]}$

Since the modulus was almost independent of the observed frequency within the range from 0.05 to $1 \mathrm{~Hz}$. in these experiments, the test frequency was kept constant at 0.5 $\mathrm{Hz}$. and the initial static strain was changed.

5-1 Experimental Results and Discussion for Looped Pile Carpets

In general, if a carpet is assumed to te made up of a fiber phase and an open air phase, its mechanical properties are considered dependent on the following factors, (1): volume fraction of the fiter phase, $(2)$ : original shape of pile and (3): pile yarn material.

Since pi'e yarn material of all looped pile carpets in this experiments is Nylon-6, five samp'es having approximately same (pile weight $/ l_{c}$ ) values but having different original shapes of looped pile due to the difference of treatment were picked up from Table 1.

Dependence of apparent complex dynamic modulus on static strain for these five looped pile carpets is shown in Fig. 7 after divided into three groups such as yarn dyed, carpet dyed and false-twisted. The difference between these dependences may be derived from the difference in original loop shape and deformed mode corresponding to loop 
shape, because (pile weight $/ l_{c}$ ) value for these carpets are much the same and pile yarn materials are all the same. Namely, the deformed mode of SP-L or P-SP-L having greater value of $\mathrm{S} / \mathrm{H}$ is that of Fig. 1-(f). In this case, jammed configuration on inter-pile yarn is not found as shown in Fig. 5, even when the static strain is increased.

On the countrary, the deformed mode of L or P-S and B samples having smaller values of $\mathbf{S} / \mathbf{H}$ is that of Fig. 1-(h) and 1-(i), respectively. In these cases, as shown in Fig. 5, jammed configuration on inter-pile yarns exists, and packing of constituent fibers advances with increasing strain. Consequently, the difference between the above mentioned dependences shown in Fig. 7 may be because of the difference of original loop shapes and deformed modes corresponding to loop shapes.

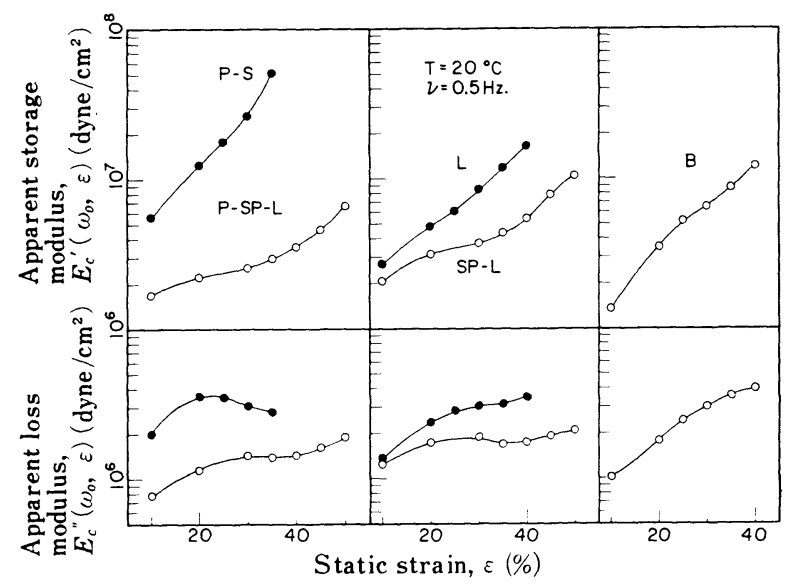

Fig. 7 Static strain dependence of apparent complex dynamic modulus for five looped pile carpets with approximately the same pile weight

From the stand point of floor absorber, the carpet having higher $E c^{\prime}$ and $E c^{\prime \prime}$ seems to be better in quality. ${ }^{[9,10]} \mathrm{As}$ seen in Fig. 7, the complex dymanic modulus differs among these carpets, which were made of the same fiber material and with the same pile weight. This shows that the looped pile carpet liable to jamm on inter-pile yarns is much more advisiable for carpets.

Effects of interaction of pile yarns at high strain can be observed in apparent compressive stress-strain curves of the carpets.

Fig. 8 shows the results of static experiment by an Instron type tester with a cylindrical compressive plate, $5 \mathrm{~cm}^{2}$ wide, and carried out at a fixed compressive speed of $5 \mathrm{~mm} /$ min. in $20{ }^{\circ} \mathrm{C}$ and $65 \%$ R.H. atmosphere. There, the vertical axis represents the value of (pick-up compressive force)/(area of the compressive plate) in place of the apparent compressive stress because the pile part behaves like a material having nearly Poisson's ratio 0 . Also, the horizontal axis repersents the compressive strain, taking $l_{c}$ as its

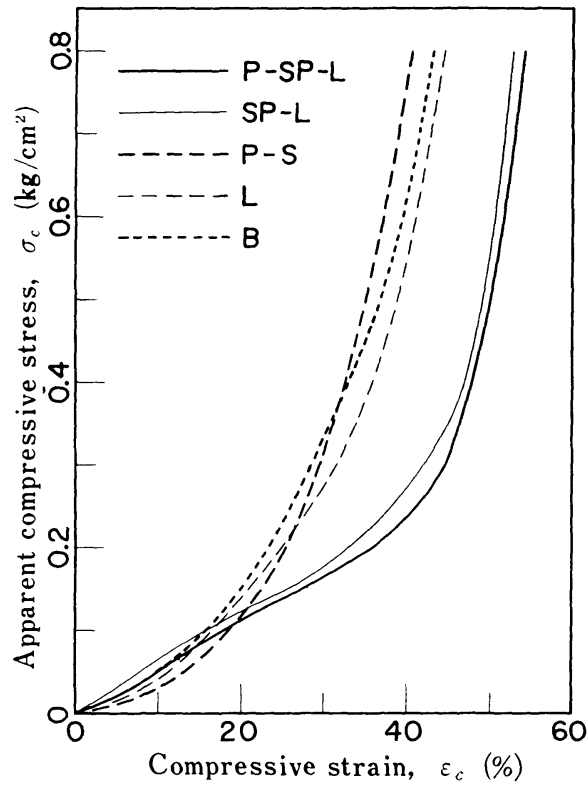

Fig. 8 Apparent compressive stress-strain curves for five looped pile carpets with approximately the same pile weight

original length, because $l_{c}$ differs among sample carpets as listed in Table 1.

At low strain, shapes of $\sigma_{c}-\varepsilon_{r}$. curves differ somewhat between SP-L or P-SP-L group having higher $\mathrm{S} / \mathrm{H}$ ratio and $\mathrm{S}, \mathrm{P}-\mathrm{L}$ or $\mathrm{B}$ group having lower $\mathrm{S} / \mathrm{H}$ ratio. The former goes up rather fast with increasing strain than the latter.

But at high strain the former group shifts to the high strain side than that of the latter.

When the pile weight varies among sample carpets, the compressive behaviors will be changed, since the volume fraction of fiber phase is different. From this reason the factor $\sigma_{c} /\left(\right.$ pile weight $/ l_{c}$ ) was adopted instead of $\sigma_{c}$ to compare the compressive behaviors of these carpets. In carpets consisting textured yarn made by stuffer box process, $\mathrm{S} / \mathrm{H}$ ratio in loop becomes larger with increasing crimp angle, and $\sigma_{c} /\left(\right.$ pile weight $\left./ l_{c}\right)-\varepsilon_{c}$ curve under high compression shifts to the higher strain side with increasing $\mathrm{S} / \mathrm{H}$. However at low strain the load-compression curves of carpets having greater crimp angle go up rather fast with increase of strain than those with smaller crimp angle.

Moreover, the static compressive behavior of sample B having high degree of fiber-distribution as shown in Fig. 2 may be considered to show clearly the effect of distribution of constituent fibers on $\sigma_{c} /\left(\right.$ pile weight $\left./ l_{c}\right)$ - $\varepsilon_{c}$ curve.

5-2 Experimental Results and Discussion for Model Cut

Pile Carpets

As is explained previously, in the model cut pile carpets only the visco-elastic behavior of the pile partcan be observed. So, some experiments were carried out to examine 


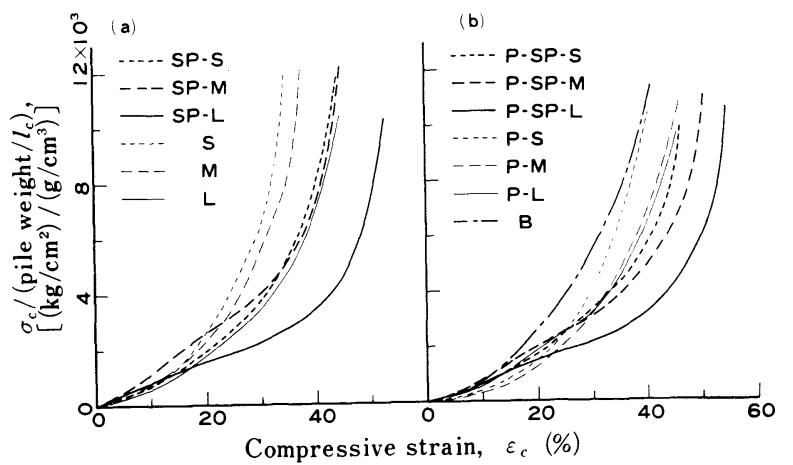

Fig. $9 \sigma_{c} /\left(\right.$ pile weight $\left./ l_{c}\right)-\varepsilon_{c}$ curves for looped pile carpets listed in Table 1

(a); carpet dyed carpets

(b); yarn dyed carpets

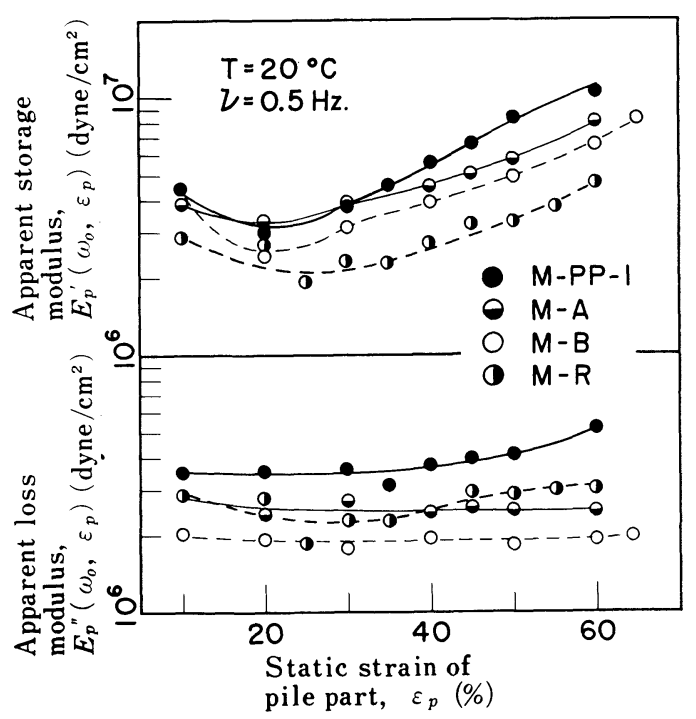

Fig. 10 Static strain dependence of apparent complex dynamic modulus for four model cut pile carpets with the same density in pile part

the dependence of fiber materials and density in pile part on viscoelastic behaviors of carpets which have the same compressive deformed mode. Figs. 10 and 11 show the results obtained for the carpets with the same density but different fiber materials, and for the carpets with the same fiber material but with different densities in pile part, respectively. As seen in these figures, the storage modulus $E p^{\prime}\left(\omega 0, \varepsilon_{p}\right)$ of all model carpets depends on the initial static strain $\varepsilon_{p}$; first $E p^{\prime}\left(\omega_{\left.0, s_{p}\right)}\right)$ decreases and then increases with strain. This may be because of the buckling of pile yarns, ${ }^{[11]}$ caused by the fact that the lower parts of pile yarns in the model carpets are fixed more firmly between metallic base pieces than commercially available carpets, and that the axes of the pile yarns are approximately perpendicular to the upper surface of the base part.

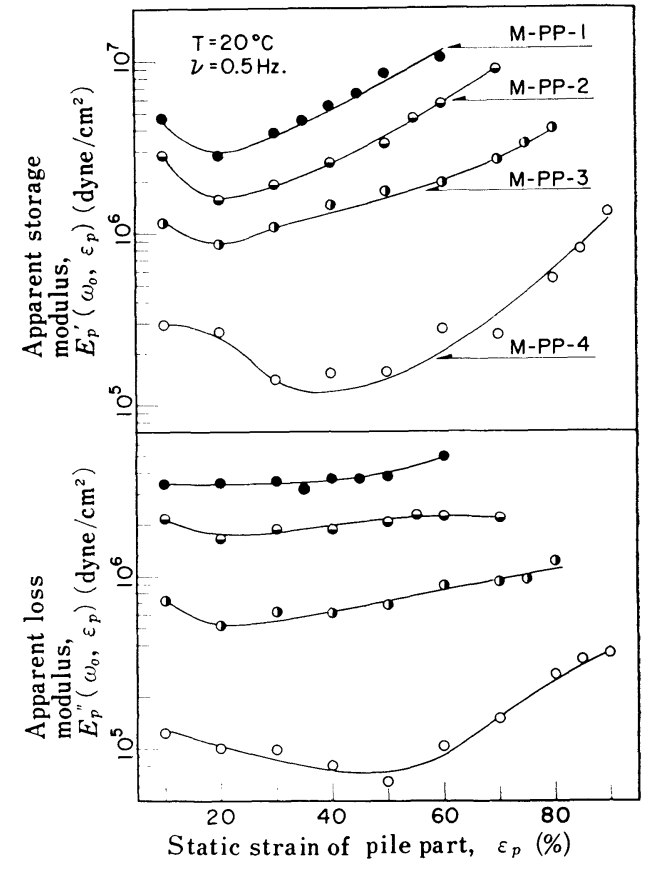

Fig. 11 Static strain dependence of apparent complex dynamic modulus for polypropylene model cut pile carpets with different density in pile part

Since the model carpets are made to have different apparent densities in pile part as listed in Table 2, the volume fraction $\dot{q}_{r}$ of fiber phase in pile part differs among carpets. So, even when the same initial static strain is applied to these carpets, $\dot{\rho}_{j}$ differs among these carpets. Therefore, $\phi_{f}$ was calculated on the assumption that the carpets behaved as a material with Poisson ratio 0 , and that the volume-decrease of the carpet when compressed took place only in the open air phase.

When the volume of pile part under $\varepsilon_{p}=0, V p(0)$ is assumed to be composed of $V_{f}$, volume of fiber phase, and $V_{a}$, volume of open air phase, and $\omega_{f}$ and $\left(_{a}\right.$, the weight of each phase, respectively, the apparent density of pile part $\omega_{n, p}$, is given by, when $\omega_{n}$ is neglected,

$$
\rho_{a, p}=\begin{aligned}
& w_{f}+w_{a} \\
& V_{f}+V_{a}
\end{aligned}=\begin{gathered}
w_{f} \\
V_{p}(0)
\end{gathered}
$$

From these assumptions, the volume of pile part under $\varepsilon_{p}, V_{p}\left(\varepsilon_{p}\right)$, reduces to $V_{p}(0)\left(1-\varepsilon_{p}\right)$. $\phi_{f}\left(\varepsilon_{p}\right)$ under this condition is given by,

$$
\phi_{f}\left(\varepsilon_{p}\right)=\begin{gathered}
V_{f} \\
V_{p}(0)\left(1-\varepsilon_{p}\right)
\end{gathered}=\frac{\rho_{a, p}}{\rho_{f}\left(1-\varepsilon_{p}\right)} \cdots(4)
$$

Thus, $\dot{\rho}_{f}\left(\varepsilon_{p}\right)$ is related to $\rho_{a}, p$ and $\rho_{j}$.

On four polypropylene model carpets having the lowest fiber density $\dot{\rho}_{f}\left(\varepsilon_{p}\right)-\xi_{p}$ curves were calculated from Eq. 


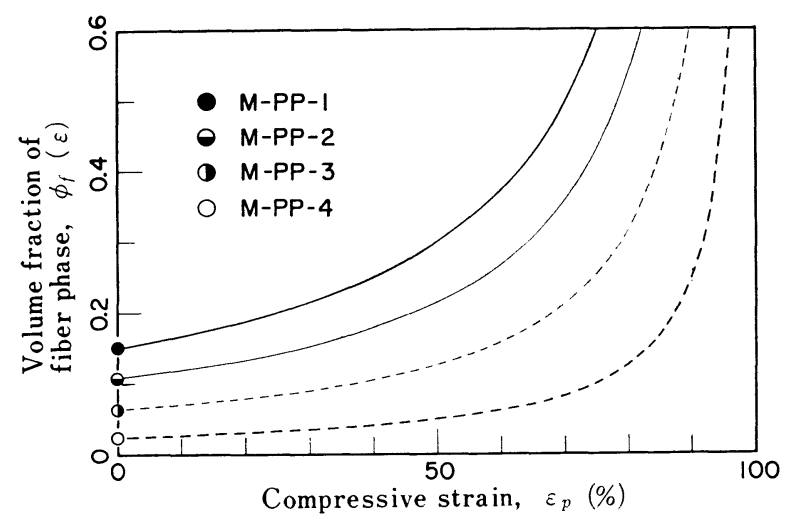

Fig. 12 Compressive strain dependence of volume fraction of fiber phase for four model cut pile carpets

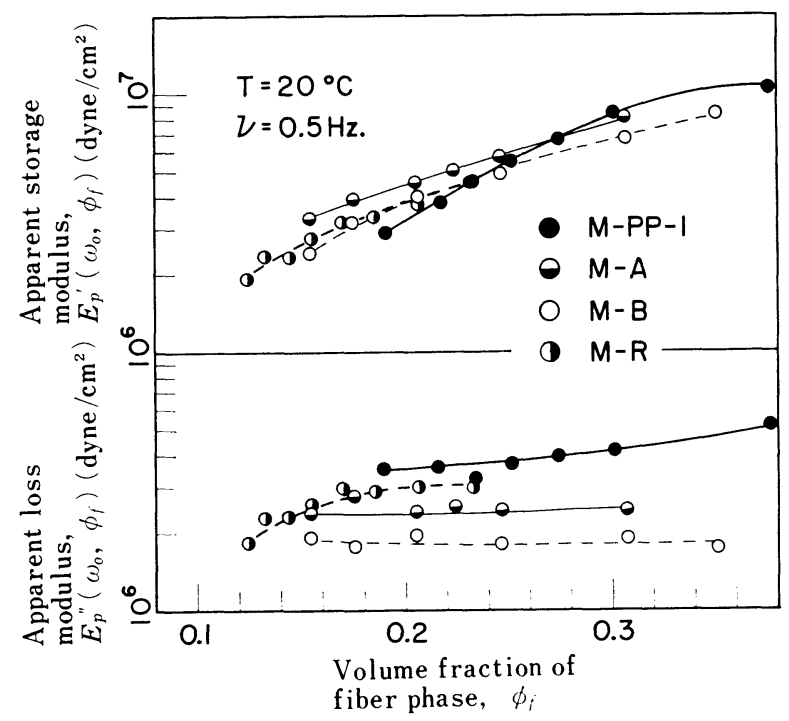

Fig. 13 Volume fraction of fiber phase dependence of apparent complex dynamic modulus for four model cut pile carpets with different pile yarns

(4) and shown in Fig. 12.

In order to reach $i_{t}\left(\varepsilon_{p}\right)=0.5$, even M-PP-1 sample having the highest value of $\|_{\mu},{ }_{p}$ at $\varepsilon_{p}=0 \%$, must ce compressed with as high strain as approximately $70 \%$. $a_{a}, p$ for many commercially available carpets is roughly equal to $0.12 \mathrm{~g} / \mathrm{cm}^{3}$.

Figs. 13 and 14 show the results calculated by Eq. (4),

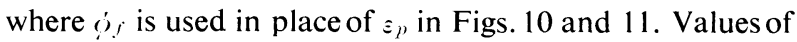
$E p^{\prime}\left(\omega_{0}, \xi\right)$ at low strain where they decrease with strain, were omitted at replotting in Figs. 13 and 14.

In case of the carpets made of different fiber materials, as shown in Fig. 13, the effect of different fiber materials on $E p^{\prime}$ is not found and the dependence of $E p^{\prime}$ on the vo-

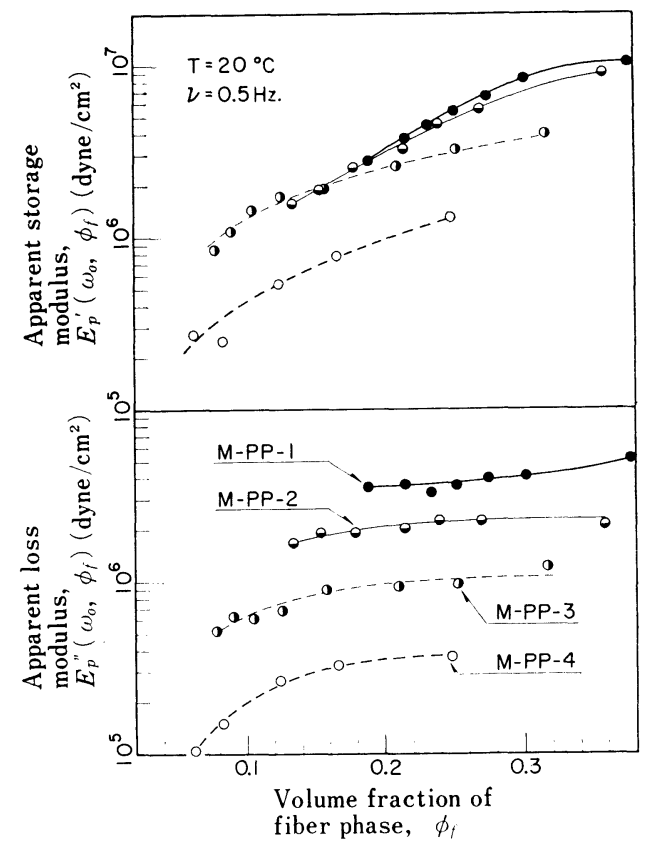

Fig. 14 Volume fraction of fiber phase dependence of apparent complex dynamic modulus for four polypropylene model cut pile carpets with different apparent density of pile part

lume fraction of fiber phace is commonly observed. On the other hand, the dependence of $E p^{\prime \prime}$ on if rather differs among different pile yarn materials. These differences may be caused by the effect of external friction for inter-pile yarns.

On the contrary, with the carpets made of the same material, $E p^{*}$ is affected by $\iota_{a, p}$ as seen in Fig. 14. The carpet having low $\rho_{a}, p$ must te compressed with greater static strains as shown in Fig. 12 to give the same value as M-PP-1 which has large $\omega_{a},{ }_{p}$. In this case, the pile yarn is strained heavily. Accordingiy, even when values of $\rho_{f}$ for carpets in Fig. 14 had the same value, $E p^{*}$ are affected by changes in fiker packing state and by the deformed state. Especially, $E_{p}{ }^{\prime \prime}$ is influenced by the difference of fibers packing state, and increases with $\rho_{a},,_{p}$ which is determined when the model carpet has been formed probably because of the external friction between inter-fibers.

When $\rho_{a},{ }_{1}$ is nearly equal to $0.12 \mathrm{~g} / \mathrm{cm}^{3}, E p^{\prime}$ may not be affected by ${ }^{\prime} a, \ldots$.

\section{Conclusions}

Various looped pile carpets having different loop shapes or degrees of fiber distribution in pile part were prepared by changing the manufacturing processes. Also, model cut pile carpets having the same apparent density ${ }_{0}{ }_{a},{ }_{l}$, but with different fiber materials, and those having the same fiber material but with different $\rho_{a},{ }_{p}$, were prepared by using 
an attachment. Their apparent complex dynamic modulus was observed at various compressive strain by Hysteresis loop method at a fixed frequency of $0.5 \mathrm{~Hz}$., and obtained on the assumption that the mechanical model of these carpets is the sarre as the Voigt model.

The result suggests that $E c^{*}$ is mainly related to three factors, i.e. (1): deformed mode of pile yarn, (2): volume fraction of fiter phase and (3): pile yarn material.

The following conclusions were obtained: (1) when the deformed mode of pile yarn changes, $E c^{*}$ seem to be affected by the difference in jammed configuration of inter-pile yarns under compression, (2) when deformed modes of pile yarns are the same, $E c^{\prime}$ and $E c^{\prime \prime}$ increase with $\rho_{a},{ }_{p}$. But the latter is affected more by $o_{a},{ }_{p}$ than the former. (3) when volume fractions of fiber phase $\phi_{f}$ are the same, $E c^{*}$ is not affected by pile yarn material, because $\dot{\rho}_{f}$ is generally small with many carpets. However, $E c^{\prime \prime}$ depends on shapes and surface structures of fiber materials.

\section{Literature cited}

[1] J. L. Barch, Text Res. J., 19, 355 (1949)

[2] D. W. Hadley and D. A. Preston, J. Text. Inst., 58, T194 (1967)

[3] C. H. Sturley and W. T. Westhead, ibid, 49, T538 (1958)

[4] H. Noshi, K. Hatiya and T. Tagawa, J. Text. Mach. Soc. Japan, 16, 294 (1962)

[5] S. Tsuchibayashi, Seni Kagaku, 5, 67 (1963-8)

[6] Aly El-Shiekh and S. P. Hersh, Text. Res. J., 39, 1134 (1969)

[7] K. Kimura, S. Kawabata and H. Kawai, J. Text. Mach. Soc. Japan, 23, T67 (1970) : ibid., 24, T207 (1971)

[8] T. Horino, T. Yabunaka and M. Morikawa, ibid., 23, T213 (1970)

[9] T. Horino and S. Kawanishi, J. Japan. Res. Ass. Text. End.-Uses, 9, 204 (1968)

[10] T. Horino and S. Kawanishi, ibid., 10, 213 (1969)

[11] M. Tabata and K. Nishimura, J. Text. Mach. Soc. Japan, 20, P. 26 (1967) 